Brief Report

matol 2011;36:845-850.

3. Zweegers J, Roosenboom B, van de Kerkhof PC, van den Reek JM, Otero ME, Atalay S, et al. Frequency and predictors of a high clinical response in patients with psoriasis on biological therapy in daily practice: results from the prospective, multicenter BioCAPTURE cohort. Br J Dermatol 2017; 176:786-793.

4. Strober BE, Bissonnette R, Fiorentino D, Kimball AB, Naldi $L$, Shear $N H$, et al. Comparative effectiveness of biologic agents for the treatment of psoriasis in a real-world setting: results from a large, prospective, observational study (Psoriasis Longitudinal Assessment and Registry [PSOLAR]). J Am Acad Dermatol 2016;74:851-861.e4.

5. Menting SP, Sitaram AS, Bonnerjee-van der Stok HM, de
Rie MA, Hooft L, Spuls PI. Drug survival is not significantly different between biologics in patients with psoriasis vulgaris: a single-centre database analysis. Br J Dermatol 2014;171: 875-883.

6. van Lümig PP, van de Kerkhof PC, Boezeman JB, Driessen $\mathrm{RJ}$, de Jong EM. Adalimumab therapy for psoriasis in real-world practice: efficacy, safety and results in biologicnaïve vs. non-naïve patients. J Eur Acad Dermatol Venereol 2013;27:593-600.

7. Shear NH, Hartmann M, Toledo-Bahena $M$, Katsambas A, Connors L, Chang Q, et al.; REALITY investigators. Longterm efficacy and safety of infliximab maintenance therapy in patients with plaque-type psoriasis in real-world practice. Br J Dermatol 2014;171:631-641.

\title{
Senile Purpura: Clinical Features and Related Factors
}

\author{
Soo Ick Cho, Ji Won Kim, Gyeongyeon Yeo ${ }^{1}$, Dongmuk Choi ${ }^{1}$, Junggyo Seo ${ }^{1}$, Hyun-Sun Yoon ${ }^{2}$, \\ Jin Ho Chung
}

Department of Dermatology, Seoul National University College of Medicine, ${ }^{1}$ Seoul National University College of Medicine, ${ }^{2}$ Department of Dermatology, SMG-SNU Boramae Medical Center, Seoul, Korea

\section{Dear Editor:}

Senile or actinic purpura commonly presents as purpuric macules and patches on the sun-exposed skin in elderly individuals. The prevalence of senile purpura is approximately $10 \%$ in elderly individuals ${ }^{1}$. Senile purpura is considered a consequence of skin aging primarily attributable to photodamage and is often called dermatoporosis ${ }^{2}$. Although it is a common and clinically important condition, only a few studies have described in detail the clinical features or factors related to senile purpura ${ }^{3}$.

We performed a cross-sectional study using questionnaires to assess the clinical features and other factors related to senile purpura at Seoul National University Hospital (SNUH) and 20 local senior welfare centers between October and December 2017. The study protocol was approved by the Institutional Review Board of SNUH (IRB no. 1708-137-879), and written informed consent was obtained from all participants.

Patients enrolled in the study were asked whether they have/had senile purpura. Using a digital thickness micrometer (Mitutoyo Corporation, Kanagawa, Japan), double fold of skin thickness was measured at the level of the extensor area of the right lower arm $15 \mathrm{~cm}$ away from the elbow.

\footnotetext{
Received October 18, 2018, Accepted for publication November 1, 2018
}

Corresponding author: Jin Ho Chung, Department of Dermatology, Seoul National University Hospital, 101 Daehak-ro, Jongno-gu, Seoul 03080, Korea. Tel: 82-2-2072-2410, Fax: 82-2-742-7344, E-mail: jhchung@snu.ac.kr ORCID: https://orcid.org/0000-0002-0582-6392

This is an Open Access article distributed under the terms of the Creative Commons Attribution Non-Commercial License (http://creativecommons.org/ licenses/by-nc/4.0) which permits unrestricted non-commercial use, distribution, and reproduction in any medium, provided the original work is properly cited.

Copyright $($ The Korean Dermatological Association and The Korean Society for Investigative Dermatology 
We used 2 questionnaires in this study - the first to assess the epidemiology and the second to assess other factors related to senile purpura. The epidemiological questionnaire included information regarding present lesions or a history of senile purpura, age of onset, frequency of development of lesions, persistence, sites of lesions, seasonal variations, inciting factors, inconvenience, and intent-totreat analysis.

The questionnaire regarding other factors related to senile purpura included the patient's medical history, lifestyle factors, and sun exposure-related factors, skin care habit and occupational history. Diagnosis of current lesions was confirmed based on the history and the current medications administered. The questionnaire assessing sun exposure-related factors was a modified version obtained from Zhu et al. ${ }^{4}$

The IBM SPSS software version 23.0 (IBM Corp., Armonk, NY, USA) was used for statistical analysis. The Pearson's chi-square test, the Fisher exact test, or the linear association method was used to compare categorical data. The Student's t-test or the Mann-Whitney $U$ test was used for intergroup comparisons of continuous variables. Binary logistic regression was performed to assess the other factors related to senile purpura. Patient-related factors that showed $p<0.10$ using univariate logistic regression analysis were subjected to multivariate logistic regression analysis. A $p$-value $<0.05$ was considered statistically significant.

Among the patients investigated, 57 (29.5\%) reported senile purpura at the time of this study $(\mathrm{n}=27,14.0 \%)$ or a history of senile purpura $(n=30,15.5 \%)$. The mean age of onset was $71.0 \pm 10.0$ years. The most common site of purpuric lesions was the lower arms and hands $(n=55$, $96.5 \%)$, followed by the lower legs and feet $(n=30,52.6 \%)$, and the thighs $(n=20,35.1 \%)$. Approximately one-third of the patients investigated $(n=17,29.8 \%)$ reported that they almost always had senile purpura lesions. More than $50 \%$ of patients $(n=34,59.6 \%)$ reported that the purpuric lesions persisted for $<2$ weeks, whereas 11 (19.3\%) patients reported that their lesions lasted $>3$ weeks. Most patients $(n=40,70.2 \%)$ reported no seasonal clinical fluctuations. More than $50 \%$ of patients (54.4\%) reported that minor trauma was an inciting factor. Notably, 22 (38.6\%) patients did not report any inconvenience from senile purpura, whereas $13(22.8 \%)$ reported interference in their daily lives (Supplementary Table 1).

No statistically significant differences were observed in demographic characteristics between patients with senile purpura and controls (Table 1). Skin thickness in the senile purpura group was lesser than that in the control group. $(p=0.010)$ Cardiac disease, dyslipidemia, and antico- agulation treatment rates in the senile purpura group were significantly higher than those in the control group ( $p=0.003,0.018$, and $<0.001$ in each).

In terms of lifestyle, patients with senile purpura showed a higher smoking tendency $(p=0.010)$ and a lower disposition for exercise, although the difference was statistically non-significant (mild exercise, $p=0.065$; Supplementary Table 2). Other lifestyle factors did not appear to be statistically significant. No significant intergroup difference was observed in both lifetime sun exposure and other sun exposure-related factors (Supplementary Table 3).

Univariate logistic regression analysis showed 7 statistically significant factors (Table 2). Multivariate analysis showed that dyslipidemia $(p=0.003)$, anticoagulant treatment $(p=0.027)$, a history of dermatological disease $(p=$ 0.004 ), and mild exercise (none vs. $5 \sim 7$ days per week, $p=0.017$ ) demonstrated statistically significant intergroup differences.

Senile purpura is considered a self-healing cutaneous disease without sequelae. Recently, skin tears are commonly being reported and are bothersome issues in elderly patients ${ }^{5}$. Most clinical aspects of senile purpura observed in this study concurred with previous studies. Lesions were most commonly observed on the extremities, and usually disappeared within 3 weeks. Most patients did not view this condition as more than a cosmetic issue at best; how-

Table 1. Demographics and medical history of the subjects

\begin{tabular}{|c|c|c|c|}
\hline Characteristic & $\begin{array}{l}\text { Senile purpura } \\
\quad(n=57)\end{array}$ & $\begin{array}{l}\text { Control } \\
(n=136)\end{array}$ & $p$-value \\
\hline Sex (male/female) & $27 / 30$ & $46 / 88^{*}$ & 0.090 \\
\hline Age (yr) & $78.2 \pm 6.6$ & $77.5 \pm 6.2$ & 0.524 \\
\hline Height $(\mathrm{cm})$ & $160.2 \pm 9.5$ & $158.3 \pm 7.5$ & 0.216 \\
\hline Weight (kg) & $59.0 \pm 9.1$ & $8.5 \pm 10.2$ & 0.757 \\
\hline Body mass index $\left(\mathrm{kg} / \mathrm{m}^{2}\right)$ & $23.4 \pm 3.1$ & $23.43 \pm 3.2$ & 0.873 \\
\hline \multicolumn{3}{|c|}{ Skin double-fold thickness (mm) } & 0.010 \\
\hline$<4$ & 18 (31.6) & $28(20.6)$ & \\
\hline $4 \sim 6$ & $23(40.4)$ & $62(45.6)$ & \\
\hline$>6$ & $9(15.8)$ & $46(33.8)$ & \\
\hline Previous $h$ & $3(5.3)$ & $6(4.4)$ & 0.725 \\
\hline Cardiac disease & $15(26.3)$ & $13(9.6)$ & 0.003 \\
\hline Hypertension & 37 (64.9) & $71(52.2)$ & 0.105 \\
\hline Diabetes mellitus & $15(26.3)$ & $29(21.3)$ & 0.451 \\
\hline Dyslipidemia & $24(42.1)$ & $34(25.0)$ & 0.018 \\
\hline $\begin{array}{l}\text { Anticoagulation } \\
\text { treatment }\end{array}$ & $23(44.2)^{\dagger}$ & $22(17.3)^{\ddagger}$ & $<0.001$ \\
\hline $\begin{array}{l}\text { Any dermatologic } \\
\text { disease history }\end{array}$ & $34(59.6)$ & $38(27.9)$ & $<0.001$ \\
\hline Other diseases history & $22(38.6)$ & $40(29.4)$ & 0.213 \\
\hline
\end{tabular}

Values are presented as number only, mean \pm standard deviation, or number (\%). *Two missing values exist. ${ }^{\mp}$ Five missing values exist. ${ }^{\ddagger}$ Nine missing values exist. 
Table 2. Logistic regression analysis for the related factors of senile purpura

\begin{tabular}{|c|c|c|c|c|c|}
\hline Characteristic & $\begin{array}{l}\text { Purpura/without } \\
\text { purpura (number) }\end{array}$ & $\begin{array}{l}\text { Unadjusted OR } \\
\qquad(95 \% \mathrm{Cl})\end{array}$ & $p$-value & $\begin{array}{l}\text { Adjusted OR } \\
(95 \% \mathrm{Cl})^{*}\end{array}$ & $p$-value \\
\hline \multicolumn{6}{|l|}{ Sex } \\
\hline Male & $27 / 46$ & Reference & & Reference & \\
\hline Female & $30 / 88$ & $0.581 \quad(0.309 \sim 1.091)$ & 0.091 & $1.313(0.309 \sim 5.578)$ & 0.712 \\
\hline \multicolumn{6}{|l|}{ Cardiac disease } \\
\hline No & $42 / 123$ & Reference & & Reference & \\
\hline Yes & $15 / 13$ & $3.379(1.487 \sim 7.681)$ & $<0.001$ & $1.930(0.585 \sim 6.366)$ & 0.280 \\
\hline \multicolumn{6}{|l|}{ Dyslipidemia } \\
\hline No & $33 / 102$ & Reference & & Reference & \\
\hline Yes & $24 / 34$ & $2.182(1.135 \sim 4.194)$ & 0.019 & $4.088(1.598 \sim 10.458)$ & 0.003 \\
\hline \multicolumn{6}{|l|}{ Anticoagulant treatment } \\
\hline No & 29/105 & Reference & & Reference & \\
\hline Yes & $23 / 22$ & $3.785(1.852 \sim 7.735)$ & $<0.001$ & $3.052(1.138 \sim 8.186)$ & 0.027 \\
\hline \multicolumn{6}{|c|}{ Any dermatologic disease history } \\
\hline No & $23 / 98$ & Reference & & Reference & \\
\hline Yes & $34 / 38$ & $3.812(1.994 \sim 7.290)$ & $<0.001$ & $3.586(1.490 \sim 8.633)$ & 0.004 \\
\hline \multicolumn{6}{|c|}{ Skin double fold thickness (mm) } \\
\hline$>6$ & $9 / 46$ & Reference & & Reference & \\
\hline $4 \sim 6$ & $23 / 62$ & $1.896(0.802 \sim 4.480)$ & 0.145 & $2.115(0.719 \sim 6.223)$ & 0.174 \\
\hline$<4$ & $18 / 28$ & $3.286(1.299 \sim 8.310)$ & 0.012 & $2.436(0.735 \sim 8.077)$ & 0.146 \\
\hline \multicolumn{6}{|l|}{ Smoking (pack year) } \\
\hline Non-smoker & $30 / 98$ & Reference & & Reference & \\
\hline Less than 20 & $9 / 14$ & $2.100(0.827 \sim 5.333)$ & 0.119 & $2.555(0.539 \sim 12.119)$ & 0.237 \\
\hline More than 20 & $17 / 22$ & $2.524(1.188 \sim 5.363)$ & 0.016 & $4.025(0.718 \sim 22.554)$ & 0.113 \\
\hline \multicolumn{6}{|l|}{ Mild exercise activity } \\
\hline None & $15 / 23$ & Reference & & Reference & \\
\hline $1 \sim 4$ days per week & $13 / 25$ & $0.797(0.313 \sim 2.029)$ & 0.635 & $0.424(0.122 \sim 1.478)$ & 0.178 \\
\hline $5 \sim 7$ days per week & $29 / 88$ & $0.505(0.233 \sim 1.096)$ & 0.084 & $0.262(0.088 \sim 0.783)$ & 0.017 \\
\hline
\end{tabular}

OR: odd ratio, $\mathrm{Cl}$ : confidence interval. *Adjusted for age, sex and all variables above mentioned.

ever, $>20 \%$ of patients reported that senile purpura lesions interfered with their daily life.

Senile purpura is often called actinic purpura owing to its association with chronic sun exposure ${ }^{6}$. Interestingly, we did not observe any distinct correlation between senile purpura and a history of sun exposure. Notably, in this study, medical and lifestyle factors were significantly associated with senile purpura. Anticoagulant therapy frequently induces purpura and is also an independent risk factor for dermatoporosis ${ }^{7}$. Evidence regarding the association between dyslipidemia and senile purpura is limited. It can be assumed that dyslipidemia might cause dysfunction or inflammation of cutaneous vessels ${ }^{8,9}$. The association between senile purpura and a history of dermatological disease might be explained by exposure to topical or systemic corticosteroids, which is a known risk factor for dermatoporosis ${ }^{2,10}$. Multivariate analysis showed that lack of exercise is a significant factor related to senile purpura. Previous studies have reported that exercise is associated with increased skin thickness ${ }^{10}$.

Limitations of our study: 1) The cross-sectional design of this study did not allow assessment of the cause-and-effect relationship; 2) This was a questionnaire-based study; thus, medical history was not obtained from the medical record system and recall bias could have potentially interfered in the questionnaires.

In conclusion, this study suggests that current medication status including dyslipidemia or anticoagulants, a history of dermatological disease, and lack of exercise could potentially serve as factors related to the development of senile purpura. Further studies are required to elucidate the pathophysiology of senile purpura.

\section{SUPPLEMENTARY MATERIALS}

Supplementary data can be found via http://anndermatol. org/src/sm/ad-31-472-s001.pdf.

\section{CONFLICTS OF INTEREST}

The authors have nothing to disclose. 


\section{ORCID}

Soo Ick Cho, https://orcid.org/0000-0003-3414-9869

Ji Won Kim, https://orcid.org/0000-0001-6982-9608

Gyeongyeon Yeo, https://orcid.org/0000-0002-7545-8708

Dongmuk Choi, https://orcid.org/0000-0002-0368-2129

Junggyo Seo, https://orcid.org/0000-0001-7478-7051

Hyun-Sun Yoon, https://orcid.org/0000-0003-1401-2670

Jin Ho Chung, https://orcid.org/0000-0002-0582-6392

\section{REFERENCES}

1. Reszke R, Pełka D, Walasek A, Machaj Z, Reich A. Skin disorders in elderly subjects. Int J Dermatol 2015;54:e332e338.

2. Kaya G, Saurat JH. Dermatoporosis: a chronic cutaneous insufficiency/fragility syndrome. Clinicopathological features, mechanisms, prevention and potential treatments. Dermatology 2007;215:284-294.

3. McKnight B, Seidel R, Moy R. Topical human epidermal growth factor in the treatment of senile purpura and the prevention of dermatoporosis. J Drugs Dermatol 2015;14: 1147-1150.

4. Zhu GA, Raber I, Sakshuwong S, Li S, Li AS, Tan C, et al. Estimation of individual cumulative ultraviolet exposure using a geographically-adjusted, openly-accessible tool. BMC Dermatol 2016;16:1.

5. Vandervord JG, Tolerton SK, Campbell PA, Darke JM, Loch-Wilkinson AM. Acute management of skin tears: a change in practice pilot study. Int Wound J 2016;13:59-64.

6. Rallis TM, Bakhtian S, Pershing LK, Krueger GG. Effects of $0.1 \%$ retinoic acid on Bateman's actinic purpura. Arch Dermatol 1995;131:493-495.

7. Saurat JH, Mengeaud V, Georgescu V, Coutanceau C, Ezzedine K, Taïeb C. A simple self-diagnosis tool to assess the prevalence of dermatoporosis in France. J Eur Acad Dermatol Venereol 2017;31:1380-1386.

8. Chen W, Jump DB, Grant MB, Esselman WJ, Busik JV. Dyslipidemia, but not hyperglycemia, induces inflammatory adhesion molecules in human retinal vascular endothelial cells. Invest Ophthalmol Vis Sci 2003;44:5016-5022.

9. Keulen ET, Schaper NC, Houben AJ, van Lin JM, Lutgens I, Rijkers K, et al. Reduced structural and functional skin capillaries in familial combined hyperlipidemia affected men, associated with increased remnant-like lipoprotein cholesterol levels. Atherosclerosis 2002;163:355-362.

10. Whitmore SE, Levine MA. Risk factors for reduced skin thickness and bone density: possible clues regarding pathophysiology, prevention, and treatment. J Am Acad Dermatol 1998;38:248-255. 
Supplementary Table 1. Clinical features of 57 senile purpura patients

\begin{tabular}{|c|c|c|}
\hline Characteristic & Category & Number (\%) \\
\hline \multirow[t]{6}{*}{ Site* } & Face & $7(12.3)$ \\
\hline & Trunk & $3(5.3)$ \\
\hline & Upper arms & $11(19.3)$ \\
\hline & Lower arms and hands & $55(96.5)$ \\
\hline & Thigh & $20(35.1)$ \\
\hline & Lower legs and feet & $30(52.6)$ \\
\hline \multirow[t]{5}{*}{ Frequency } & Almost always & $17(29.8)$ \\
\hline & Twice a month or more & $6(10.5)$ \\
\hline & Once a month & $4(7.0)$ \\
\hline & Once in two or three months & $8(14.0)$ \\
\hline & Not answered & $22(38.6)$ \\
\hline \multirow[t]{5}{*}{ Persistence } & More than 3 weeks & $11(19.3)$ \\
\hline & $2 \sim 3$ weeks & $4(7.0)$ \\
\hline & $1 \sim 2$ weeks & $21(36.8)$ \\
\hline & Less than 1 week & $13(22.8)$ \\
\hline & Not answered & $8(14.0)$ \\
\hline \multirow[t]{6}{*}{ Seasonal variation } & Always (no variation) & $40(70.2)$ \\
\hline & Spring & $2(3.5)$ \\
\hline & Summer & $7(12.3)$ \\
\hline & Autumn & $0(0.0)$ \\
\hline & Winter & $2(3.5)$ \\
\hline & Not answered & $6(10.5)$ \\
\hline \multirow[t]{5}{*}{ Inducing factor } & Indoor activity & $8(14.0)$ \\
\hline & Outdoor activity & $8(14.0)$ \\
\hline & Minor trauma & $31(54.4)$ \\
\hline & No & $13(22.8)$ \\
\hline & Not answered & $1(1.8)$ \\
\hline \multirow[t]{3}{*}{ Inconvenience } & Uncomfortable daily life & $13(22.8)$ \\
\hline & Cosmetic problem & $22(38.6)$ \\
\hline & None & $22(38.6)$ \\
\hline
\end{tabular}

*Duplicate responses allowed. 
Brief Report

Supplementary Table 2. Life style factors in senile purpura group and control group

\begin{tabular}{|c|c|c|c|c|}
\hline Characteristic & Category & Senile purpura $(n=57)$ & Control $(n=136)$ & $p$-value \\
\hline \multirow[t]{3}{*}{ Smoking } & Non-smoker & $30(52.6)$ & $98(72.1)$ & 0.010 \\
\hline & $<20$ pack year & $9(15.8)$ & $14(10.3)$ & \\
\hline & $\geq 20$ pack year & $17(29.8)$ & $22(16.2)$ & \\
\hline \multirow[t]{2}{*}{ Drinking } & Frequency & $0.68 \pm 1.38$ & $0.90 \pm 1.90$ & 0.431 \\
\hline & Dose & $1.03 \pm 2.38$ & $1.18 \pm 2.74$ & 0.717 \\
\hline \multirow[t]{3}{*}{ Intense exercise activity } & None & $56(98.2)$ & $125(91.9)$ & 0.096 \\
\hline & $1 \sim 4$ days per week & $1(1.8)$ & $9(6.6)$ & \\
\hline & $5 \sim 7$ days per week & $0(0)$ & $2(1.5)$ & \\
\hline \multirow[t]{3}{*}{ Moderate exercise activity } & None & $45(78.9)$ & $105(77.2)$ & 0.963 \\
\hline & $1 \sim 4$ days per week & $5(8.8)$ & $16(11.8)$ & \\
\hline & $5 \sim 7$ days per week & $7(12.3)$ & $15(11.0)$ & \\
\hline \multirow[t]{3}{*}{ Mild exercise activity } & None & $15(26.3)$ & $23(16.9)$ & 0.065 \\
\hline & $1 \sim 4$ days per week & $13(22.8)$ & $25(18.4)$ & \\
\hline & $5 \sim 7$ days per week & $29(50.9)$ & $88(64.7)$ & \\
\hline \multirow[t]{5}{*}{ Moisturizer habit } & Never & $34(59.6)$ & $86(63.2)$ & 0.507 \\
\hline & Rarely & $2(3.5)$ & $5(3.7)$ & \\
\hline & Sometimes & $6(10.5)$ & $19(14.0)$ & \\
\hline & Often & $4(7.0)$ & $6(4.4)$ & \\
\hline & Always & $11(19.3)$ & $20(14.7)$ & \\
\hline \multirow[t]{5}{*}{ Skin irritating habit } & Never & $17(29.8)$ & $29(21.3)$ & 0.926 \\
\hline & Rarely & $3(5.3)$ & $10(7.4)$ & \\
\hline & Sometimes & $9(15.8)$ & $41(30.1)$ & \\
\hline & Often & $20(35.1)$ & $40(29.4)$ & \\
\hline & Always & $8(14.0)$ & $16(11.8)$ & \\
\hline \multirow[t]{3}{*}{ Life time working $(y r)$} & Indoor blue-collar & $21.17 \pm 22.22$ & $24.73 \pm 23.60$ & 0.353 \\
\hline & Outdoor blue-collar & $7.37 \pm 14.79$ & $7.26 \pm 15.24$ & 0.966 \\
\hline & White-collar & $6.65 \pm 12.70$ & $6.18 \pm 12.41$ & 0.820 \\
\hline \multirow[t]{3}{*}{ Occupational history } & Outdoor labor & $34(59.6)$ & $77(56.6)$ & 0.698 \\
\hline & Indoor labor & $16(28.1)$ & $36(26.5)$ & 0.842 \\
\hline & Office work & 15 (26.3) & $33(24.3)$ & 0.784 \\
\hline
\end{tabular}

Values are presented as number $(\%)$ or mean \pm standard deviation. 
Supplementary Table 3. Sun exposure-related factors in senile purpura group and control group

\begin{tabular}{|c|c|c|c|c|}
\hline Characteristic & Category & Senile purpura $(n=57)$ & Control $(n=136)$ & $p$-value \\
\hline \multirow[t]{4}{*}{ Life time blistering sunburn } & None & $50(87.7)$ & $126(92.6)$ & 0.276 \\
\hline & $1 \sim 5$ & $5(8.8)$ & $7(5.1)$ & \\
\hline & $6 \sim 10$ & $2(3.5)$ & $2(1.5)$ & \\
\hline & $>10$ & $0(0.0)$ & $1(0.7)$ & \\
\hline \multirow[t]{5}{*}{ Sunscreen habit } & Never & $46(80.7)$ & $106(77.9)$ & 0.630 \\
\hline & Rarely & $2(3.5)$ & $6(4.4)$ & \\
\hline & Sometimes & $3(5.3)$ & $8(5.9)$ & \\
\hline & Often & $3(5.3)$ & $2(1.5)$ & \\
\hline & Always & $3(5.3)$ & $14(10.3)$ & \\
\hline \multirow[t]{5}{*}{ Wear a shirt with sleeves covering shoulders } & Never & $19(33.3)$ & $50(36.8)$ & 0.992 \\
\hline & Rarely & $5(8.8)$ & $4(2.9)$ & \\
\hline & Sometimes & $7(12.3)$ & $12(8.8)$ & \\
\hline & Often & $4(7.0)$ & $17(12.5)$ & \\
\hline & Always & $22(38.6)$ & $52(38.2)$ & \\
\hline \multirow[t]{5}{*}{ Stay in the shade or under an umbrella } & Never & $15(26.3)$ & $29(21.3)$ & 0.564 \\
\hline & Rarely & $1(1.8)$ & $13(9.6)$ & \\
\hline & Sometimes & $12(21.1)$ & $32(23.5)$ & \\
\hline & Often & $18(31.6)$ & $44(32.4)$ & \\
\hline & Always & $11(19.3)$ & $18(13.2)$ & \\
\hline \multirow[t]{2}{*}{ Life time sun exposure $(h)$} & Summer & $48,491.9 \pm 21,439.8$ & $52,884.8 \pm 22,165.4$ & 0.243 \\
\hline & Winter & $43,917.8 \pm 21,902.1$ & $42,612.2 \pm 23,389.2$ & 0.740 \\
\hline
\end{tabular}

Values are presented as number (\%) or mean \pm standard deviation. 